\title{
An Efficient Synthesis of Biopertinent Dihydropyrimidine (thi) one Derivatives via Three- component One-pot Synthesis Catalyzed by Tetrachlorosilane
}

\author{
T. K. Khatab*, Kh.A. M. El-Bayouki, W.M. Basyouni and \\ F. M. A. Sroor \\ Organometallic and Organometalloid Chemistry Department, \\ National Research Centre, Giza, Egypt.
}

\begin{abstract}
ERIES of dihydropyrimidine(thi)one derivatives were prepared, in $\checkmark$ good yields at room temperature by using a modified Biginelli reaction. The products were obtained through a one-pot threecomponent coupling of $\beta$-diketones, aldehydes and urea (or thiourea) in the presence of a catalytic amount of tetrachlorosilane (TCS). The effect of catalyst type, molar ratios of reactants and solvent type were also investigated; best results were obtained when TCS was employed as a catalyst and $\mathrm{CH}_{2} \mathrm{Cl}_{2}$ as a solvent at room temperature.
\end{abstract}

Keywords: Three-component, Dihydropyrimidinones, Biginelli reaction and Tetrachlorosilane.

The Biginelli reaction has been reviewed ${ }^{(1)}$. Several improved protocols for the preparation of dihydropyrimidines (DHPMs) have been reported, either by modification of the classical one-pot condensation approach itself ${ }^{(2-6)}$, or by the development of novel approach but more complex, multi-step strategies ${ }^{(7)}$. However, some of the reported methods suffer from drawbacks such as unsatisfactory yields, cumbersome product isolation procedures and environmental pollution ${ }^{(6,8-13)}$. Moreover, some of the methods are only practical for aromatic aldehydes ${ }^{(6,14)}$. Furthermore, in order to improve the efficiency of Biginelli reaction, a variety of catalysts have been reported of which $\mathrm{H}_{4} \mathrm{PMo11VO} 40^{(15)}$, Dowex-50W ${ }^{(16)}, \mathrm{H}_{3} \mathrm{PW}_{12} \mathrm{O}_{40} / \mathrm{SiO}_{2}{ }^{(17)}, \mathrm{MgBr}_{2}{ }^{(18)}$, polymer supported 4-aminoformoyldiphenylammonium triflate ${ }^{(19)}, \quad \mathrm{NaHSO}_{4} / \mathrm{SiO}_{2}{ }^{(20)}$, $\mathrm{FeCl}_{3}{ }^{(21)}, \quad \mathrm{ZrCl}_{4}{ }^{(22)}, \quad \mathrm{Cu}(\mathrm{OTf})_{2}{ }^{(23)}, \quad \mathrm{Bi}(\mathrm{OTf})_{3}{ }^{(24)}, \quad$ yutterbium triflate ${ }^{(25)}$, $\mathrm{NH}_{2} \mathrm{SO}_{3} \mathrm{H}^{(26)}$, 12-Molybdopho sphoric acid ${ }^{(27)}$, natural HEU type zeolite ${ }^{(28)}$, $\mathrm{Sr}(\mathrm{OTf})_{2}{ }^{(29)}$, covalently anchored sulfonic acid onto silica ${ }^{(30)}, \mathrm{ZrOCl}_{2} .8 \mathrm{H}_{2} \mathrm{O}^{(31)}$, silica triflate ${ }^{(32)}, \mathrm{Fe}\left(\mathrm{HSO}_{4}\right)_{3}{ }^{(33)}, \mathrm{TCICA}^{(34)}, \mathrm{PPh}_{3}{ }^{(35)}, \mathrm{CaF}_{2}{ }^{(36)}, \quad[\mathrm{bmim}] \mathrm{BF}_{4-}$ immobilized $\mathrm{Cu}(\mathrm{II})$ acetylacetonate ${ }^{(37)},[\mathrm{bmim}]\left[\mathrm{FeCl}_{4}\right]^{(38)}$, ionic liquids under ultrasound irradiation ${ }^{(39)}$, melamine trisulfonic acid ${ }^{(40)}$, silica sulfuric acid ${ }^{(41)}$ are examples.

*Corresponding author. Tel.: +20233371010; Fax: +20237601877,

E-Mail address: tamer_khatab@ hotmail.com 
Dihydropyrimidinones are significant class of organic compounds, with prominent biological activities. Several functionalized dihydropyrimidines have been found to exhibit a wide spectrum of biological effects including antiviral, antitumor, antibacterial and anti-inflammatory activities ${ }^{(42)}$. In addition, 4aryldihydropyrimidines have emerged ${ }^{(43)}$ as potent calcium channel blockers, antihypertensive, $\alpha_{1 \mathrm{a}}$-adrenergic antagonists and neuropeptide antagonists. Furthermore, dihydropyrimidinone-5-carboxylate core unit is found in many marine natural products ${ }^{(44)}$ including Batzelladine alkaloids, which are potent-HIV gp-120$\mathrm{CD}_{4}$ inhibitors. A number of novel 5- $\left(\mathrm{CF}_{3} \mathrm{CO}\right)$ dihydropyrimidine(thi)ones have been synthesized using Trifluoromethyl-1,3-diones as reactants in the Biginelli reaction promoted by $\mathrm{Me}_{3} \mathrm{SiCl}$. Thus, a set of new $\mathrm{CF}_{3}$-containing dihydropyrimidine(thi)one derivatives was obtained ${ }^{(45-47)}$.

Thiourea was also used as one of the ingredients with similar success to provide the corresponding dihydropyrimidin-2(1H)-thiones, which are also of interest for their biological activities. A novel and efficient task-specific ionic liquid synthesis of Biginelli compounds has been developed. Ionic liquid-phase bound acetoacetate reacted with (thio)ureas and various aldehydes with ionic liquid-phases supported 3,4-dihydropyrimidine-2-(thi)ones. The desired 3,4dihydropyrimidine-2-(thi)ones were easily separated from the ionic liquid-phase by trans-esterification under mild conditions in good yields and high purity ${ }^{(48)}$. An effective procedure for Biginelli's three component condensation producing 3,4-dihydropyrimidin-2(1H)-thiones by employing calcium fluoride has been carried out. In order to extend our knowledge in structure-activity relationship, all newly synthesized compounds are tested for their in vitro antibacterial and antifungal activities and the influence of some structural variations was evaluated by varying the substituent at the phenyl group in the synthesized compounds ${ }^{(49-53)}$. Therefore, a need still exists for versatile, simple and environment friendly processes whereby DHPMs may be formed under milder and practical conditions, versatile catalytic systems and thus, there is a room for further improvement toward milder reaction conditions, variations of substituents in all three components and better yields.

As a part of ongoing research program directed towards the development of new and rapid synthetic methods using silicon reagents for the construction of biologically active structural motifs ${ }^{(54)}$, it was intended, in the present work, to develop rapid, efficient and inexpensive protocol for a one-pot synthesis of dihydropyrimidine(thi)one derivatives based on the Biginelli reaction using tetrachlorosilane as heterogeneous catalyst. As compared to expensive Lewis acid catalysts, such as lanthanide chlorides and lanthanide triflates, tetrachlorosilane (TCS) is inexpensive and can be prepared very easily. The most important and salient feature of the present reaction is using very small amount of a cheap and simple chlorinated solvent. Moreover, no side products were observed in these reactions. Furthermore, the reaction can be scaled up to a multigram scale. This method allowed obtaining excellent yields of the required product in shorter reaction times as compared to those of classical methods.

Egypt. J. Chem. 56, No.4 (2013) 


\section{Results and Discussion}

1,4-Dihydropyrimidinones (DHPMs) comprise of a pyrimidine scaffold having resemblance with structures of the nucleic acid bases found in DNA and RNA. Their involvement as bases in nucleic acids has a great significance in drug design. Biginelli dihydropyrimidinone derivatives are considered as structural analogs of Monastrol, a known human kinesin Eg5 inhibitor. Racemic dihydropyrimidinone is considered to be an allosteric inhibitor of the antibody \# 7625 Eg5 and unlike taxanes, it is nontoxic to neuron cells. Monastrol is the only cell-permeable molecule currently known to specifically inhibit mitotic kinesin Eg5 and can therefore be considered as a leader for the development of new anticancer drugs ${ }^{(55 a)}$. Several marine natural products with interesting biological activities containing the dihydropyrimidine-5-carboxylate core have been isolated. Most notably among these are the Batzelladine alkaloids A and B which inhibit the binding of HIV envelope protein gp-120 to human CD4 cells and, therefore, are potential new leads for AIDS therapy ${ }^{(55 b)}$. Silica chloride is one of the most versatile and utilized catalyst for the selective construction of heterocyclic ring systems, in particular for the synthesis of 3,4dihydropyrimidinones $^{(56)}$. To the best of our knowledge, Biginelli-type reactions catalyzed by silicon reagents have been rarely reported ${ }^{(57)}$. In the present work, dihydropyrimidin-2(1H)- (thi)ones, (4) were synthesized in good yield via a three-component, one-pot reaction of aromatic aldehyde (1), 1,3-diketone (2) and urea (or thiourea) (3) in methylene dichloride as a solvent in the presence of catalytic amounts of tetrachlorosilane (TCS) (Scheme 1).
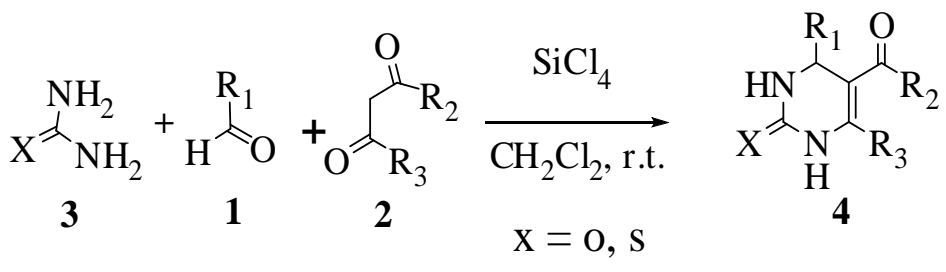

Scheme 1. Synthesis of dihydropyrimidin ones.

As a part of ongoing study to investigate the optimum conditions for the three-component Biginelli condensation catalyzed by TCS, $\mathrm{FeCl}_{3}, \mathrm{ZnCl}_{2}$ and $\mathrm{SnCl}_{2}$ in the present work was endeavored by examining the conditions required for the reaction involving thiophen-2-carbaldehyde, thiourea and ethyl acetoacetate to afford the 5-ethoxycarbonyl-6- methyl-4- (2-thienyl)3,4- dihydropyrimidin-2(1H)-thione (4aa) in some selected solvents. A summary of the results obtained is provided in Table 1. 
TABLE 1. Effect of catalysts and solvents on the yield and reaction time of the one-pot synthesis of 5-ethoxycarbonyl-6- methyl-4- (2-thienyl)-3,4-dihydropyrimidin-2 (1H)-thione(4aa).

\begin{tabular}{|c|c|c|c|c|}
\hline Entry & Catalyst & Solvent & Time (h) & Yield (\%) \\
\hline 1 & TCS & DMF & 10 & 15 \\
\hline 2 & TCS & THF & 10 & Nil \\
\hline 3 & TCS & $\mathrm{CHCl}_{3}$ & 5 & 82 \\
\hline 4 & $\mathrm{TCS}$ & $\mathrm{CH}_{2} \mathrm{Cl}_{2}$ & 3 & 89 \\
\hline 5 & $\mathrm{FeCl}_{3}$ & $\mathrm{CH}_{2} \mathrm{Cl}_{2}$ & $10, \Delta$ & 24 \\
\hline 6 & $\mathrm{ZnCl}_{2}$ & $\mathrm{CH}_{2} \mathrm{Cl}_{2}$ & $10, \Delta$ & 52 \\
\hline 7 & $\mathrm{SnCl}_{2}$ & $\mathrm{CH}_{2} \mathrm{Cl}_{2}$ & $10, \Delta$ & 58 \\
\hline
\end{tabular}

$\Delta$ heat $\left(60-70{ }^{\circ} \mathrm{C}\right)$.

Data in Table 1 revealed that, the reaction of ethylacetoacetate, thiophen-2carbaldehyde and thiourea as a model example (1 equiv-each), proceeds in the presence of tetrachlorosilane $\left(\mathrm{SiCl}_{4}\right)$ at room temperature in various solvents. Chlorinated solvents such as $\mathrm{CH}_{2} \mathrm{Cl}_{2}$ or $\mathrm{CHCl}_{3}$ were found to be effective solvents. The reaction was not compatible with solvents such as DMF (15\% yield) or THF (which completely inhibited the reaction). Thus, the best results obtained were when TCS was employed as a catalyst and $\mathrm{CH}_{2} \mathrm{Cl}_{2}$ as solvent (entry 4 , afforded the desired product in high yield ( $89 \%)$. Thus, it was prompted to explore the potential of using this protocol for the synthesis of various dihydropyrimidinones and dihydropyrimidinthiones (56-92\% yields). The reactions proceeded smoothly by stirring in chloronated solvent at room temperature and were completed within the required time. Tables 2 and 3 show the generality of the present protocol, which is equally effective for urea or thiourea and also the selected aldehyde. Under these conditions, the yields were significantly better in comparison with the classical Biginelli procedure. In all cases, the desired dihydropyrimidine-2-(thi)one were the sole products and no by-products were observed. The experimental procedure is very simple and convenient.

The results clearly indicated the generality and scope of the reaction with respect to various aromatic and hetero-aromatic aldehydes. Noteworthy to report is that data depicted in Table 2 showed that bulky aldehydes gave lower dihydropyrimidinoes yield due to their steric hindrance compared to homocyclic and hetero-aromatic aldehydes.

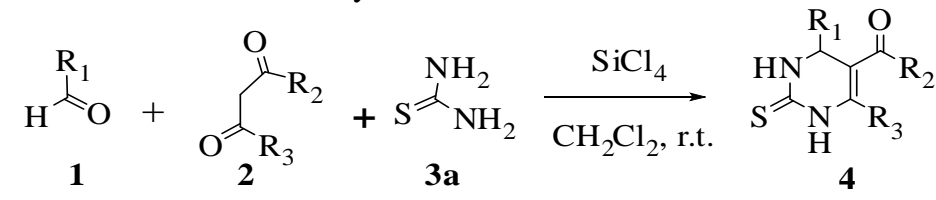

1a, $\mathrm{R}_{1}=2$-thienyl 2a, $\mathrm{R}_{2}=-\mathrm{OEt}, \mathrm{R}_{3}=-\mathrm{CH}_{3}$

1b, $\mathrm{R}_{1}=1$-naphthyl 2b, $\mathrm{R}_{2}=-\mathrm{CH}_{3}, \mathrm{R}_{3}=-\mathrm{CH}_{3}$

1c, $\mathrm{R}_{1}=2$-naphthyl 2c, $\mathrm{R}_{2}=-\mathrm{C}_{6} \mathrm{H}_{5}, \mathrm{R}_{3}=-\mathrm{CH}_{3}$

4aa, 4ab, 4ac, 4ba,

$4 \mathrm{bc}, 4 \mathrm{ca}, 4 \mathrm{cb}$

Egypt. J. Chem. 56, No.4 (2013) 
Scheme 2.

TABLE 2. Reaction of thiourea (3a) with various aldehydes and 1,3-diketones.

\begin{tabular}{|c|c|c|c|c|c|c|c|c|}
\hline \multirow{2}{*}{ Entry } & \multirow[b]{2}{*}{$\mathbf{R}_{1}$} & \multirow[b]{2}{*}{$\mathbf{R}_{2}$} & \multirow[b]{2}{*}{$\mathbf{R}_{\mathbf{3}}$} & \multirow{2}{*}{ Product } & \multirow{2}{*}{$\begin{array}{c}\text { Time } \\
\text { (h) }\end{array}$} & \multirow{2}{*}{$\begin{array}{c}\text { Yield }^{\mathrm{b}} \\
(\%)\end{array}$} & \multicolumn{2}{|c|}{$\mathbf{M p}^{\mathbf{c}}\left({ }^{\circ} \mathbf{C}\right)$} \\
\hline & & & & & & & found & reported \\
\hline 1 & 2-thienyl & $-\mathrm{OEt}$ & $-\mathrm{CH}_{3}$ & $4 \mathrm{aa}$ & 3 & 89 & $213-215$ & $215-216^{58}$ \\
\hline 2 & 2-thienyl & $-\mathrm{CH}_{3}$ & $-\mathrm{CH}_{3}$ & $4 a b$ & 4 & 86 & $222-224$ & -- \\
\hline 3 & 2-thienyl & $\mathrm{C}_{6} \mathrm{H}_{5^{-}}$ & $-\mathrm{CH}_{3}$ & $4 \mathrm{ac}$ & 5 & 81 & $235-235$ & -- \\
\hline 4 & 1-naphthyl & $-\mathrm{OEt}$ & $-\mathrm{CH}_{3}$ & $4 \mathrm{ba}$ & 6 & 79 & $218-220$ & -- \\
\hline 5 & 1-naphthyl & $\mathrm{C}_{6} \mathrm{H}_{5^{-}}$ & $-\mathrm{CH}_{3}$ & $4 \mathrm{bc}$ & 8 & 75 & 295(dec.) & -- \\
\hline 6 & 2-naphthyl & $-\mathrm{OEt}$ & $-\mathrm{CH}_{3}$ & $4 \mathrm{ca}$ & 5 & 81 & $185-186$ & $188^{59}$ \\
\hline 7 & 2-naphthyl & $-\mathrm{CH}_{3}$ & $-\mathrm{CH}_{3}$ & $4 \mathrm{cb}$ & 6 & 76 & 236 & $238-240^{59}$ \\
\hline
\end{tabular}

${ }^{\mathrm{c}}$ Melting points are uncorrected

${ }^{\mathrm{b}}$ Isolated yield

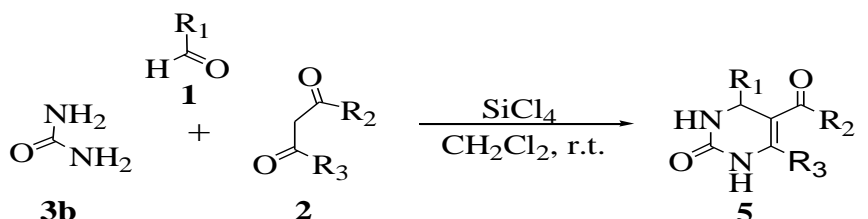

1d, $R_{1}=$ furyl 2d, 1,3-indandione

1e, $R_{1}=9$-anthryl 2e, dimedone

$5 a a, 5 a b, 5 d a, 5 b a$,

1f, $R_{1}=$ phenyl

1g, $\mathrm{R}_{1}=4-\mathrm{Cl}-\mathrm{C}_{6} \mathrm{H}_{4}$

1h, $\mathrm{R}_{1}=4-\mathrm{Br}-\mathrm{C}_{6} \mathrm{H}_{4}$

Scheme 3

TABLE 3. Reaction of thiourea (3a) with various aldehydes and 1,3-diketones.

\begin{tabular}{|c|c|c|c|c|c|c|c|c|}
\hline \multirow{2}{*}{ Entry } & \multirow{2}{*}{$\mathbf{R}_{1}$} & \multirow{2}{*}{$\mathbf{R}_{2}$} & \multirow{2}{*}{$\mathbf{R}_{\mathbf{3}}$} & \multirow{2}{*}{ Product } & \multirow{2}{*}{$\begin{array}{c}\text { Time } \\
\text { (h) }\end{array}$} & \multirow{2}{*}{$\begin{array}{c}\text { Yield }^{b} \\
(\%)\end{array}$} & \multicolumn{2}{|c|}{$\mathbf{M p}^{\mathbf{c}}\left({ }^{\circ} \mathbf{C}\right)$} \\
\hline & & & & & & & found & reported \\
\hline 1 & 2-thienyl & -OEt & $-\mathrm{CH}_{3}$ & $5 \mathrm{aa}$ & 3 & 92 & $212-213$ & $215-217^{58}$ \\
\hline 2 & 2-thienyl & $-\mathrm{CH}_{3}$ & $-\mathrm{CH}_{3}$ & $5 \mathrm{ab}$ & 4 & 88 & 220-221 & -- \\
\hline 3 & 2-furyl & -OEt & $-\mathrm{CH}_{3}$ & $5 \mathrm{da}$ & 5 & 58 & 205-206 & $206-208^{59}$ \\
\hline 4 & 1-naphthyl & -OEt & $-\mathrm{CH}_{3}$ & $5 \mathrm{ba}$ & 6 & 83 & 242-244 & $247-248^{60}$ \\
\hline 5 & 1-naphthyl & \multicolumn{2}{|c|}{ 1,3-indandione } & $5 b d$ & 9 & 56 & $236-238$ & -- \\
\hline 6 & 9-anthryl & $-\mathrm{OEt}$ & $-\mathrm{CH}_{3}$ & 5 ea & 7 & 74 & 248-249 & $251^{60}$ \\
\hline 7 & 4- $\mathrm{Cl}-\mathrm{C}_{6} \mathrm{H}_{4}-$ & \multicolumn{2}{|c|}{ demidone } & $5 \mathrm{ge}$ & 4 & 81 & 195 & $196-198^{61}$ \\
\hline 8 & 4-Br- $\mathrm{C}_{6} \mathrm{H}_{4}-$ & \multicolumn{2}{|c|}{ demidone } & 5 he & 5 & 80 & $>300$ & $>300^{62}$ \\
\hline
\end{tabular}

${ }^{\mathrm{c}}$ Melting points are uncorrected

${ }^{\mathrm{b}}$ Isolated yield

Recently, it has been reported that using thiourea and ethylacetoacetate increased reaction time for affording some dihydropyrimidinethiones, reduced the efficiency of Biginelli reaction. This is related to the low nucleophilic 
property of thiourea compared to urea at intermediate state ${ }^{(62)}$. However, it was found in the present work the desired dihydropyrimidinethiones (4) were obtained in $3-8 \mathrm{~h}$ and $75-89 \%$ yield as shown in Table 2. In comparison the dihydropyrimidinones (5) were formed in 3-9 $\mathrm{h}$ with $56-92 \%$ yield as given in Table 3.

The structures of the obtained dihydropyrimidinones and dihydropyrimidinthiones were confirmed by spectroscopic methods and elemental analysis Thus, the structure of 5-acetyl -6-methyl-4-(2-thienyl)-3,4-dihydropyrimidin$2(1 \mathrm{H})$-thione $(4 \mathrm{ab})$ was elucidated by its IR spectrum which showed peaks at $v=$ 3274,3167 and $1662 \mathrm{~cm}^{-1}$ corresponding to $(2 \mathrm{NH})$ and $\alpha, \beta$-unsaturated carbonyl groups, respectively; ${ }^{1} \mathrm{H}$ NMR spectrum revealed clearly two signals for exchangeable protons of $(2 \mathrm{NH})$ at $\delta_{\mathrm{H}}=9.85-10.41$, multiplet for three aromatic protons at $\delta_{\mathrm{H}}=6.94-7.41$, singlet signals for the protons $\underline{\mathrm{CHNH}}$ at $\delta_{\mathrm{H}}=5.30$, two singlet signals for $2 \mathrm{CH}_{3}$ at $\delta_{\mathrm{H}}=2.31,2.21 \mathrm{ppm} ;{ }^{13} \mathrm{C} \mathrm{NMR}$ spectrum for $(4 \mathrm{ab})$ showed two characteristic signals at $\delta=196.30,184.20$, for $\mathrm{C}=\mathrm{O}$ and $\mathrm{C}=\mathrm{S}$, at $150.20,113.52$ for $\mathrm{C}=\mathrm{C}$ in pyrimidine ring, at $139.3,126.5,125,123.4$ for thiophene ring carbons, at 53.60 charcteristic signal for saturated carbon in pyrimidine ring, at $22.70,18.22$ for two carbons in $2 \mathrm{CH}_{3}$.

A plausible mechanism for this reaction involves the formation of the enolizable 1,3-diketone and polarized aldehyde by joining with two molecules of $\mathrm{SiCl}_{4}$; these two active intermediates (I) interact with each other to form intermediate (II) which easily condenses with urea (thiourea) to form pyrimidine ring (III) as follows:

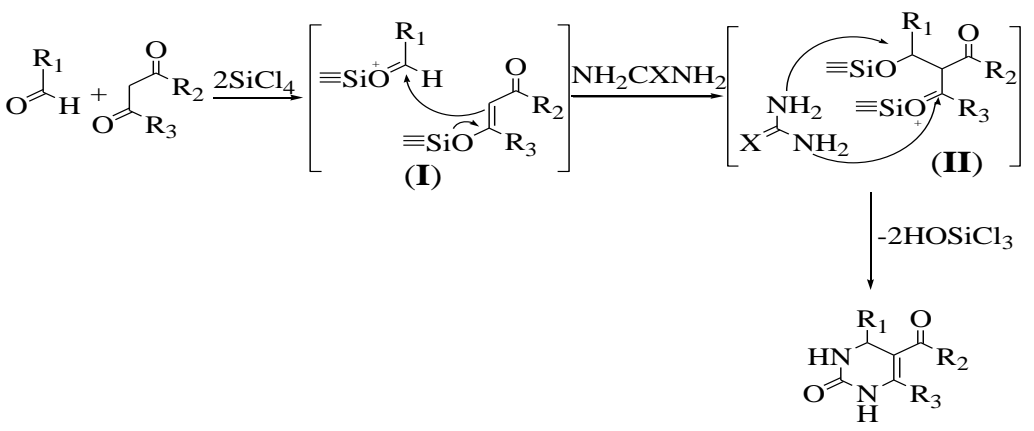

Scheme 4. Suggested mechanism.

(III)

\section{Conclusion}

In conclusion, the present protocol provides an efficient and improved modification of the Biginelli reaction. Mild reaction conditions, ease of workup, high-satisfactory yields, and stability and recyclability of tetrachlorosilane reagent are features of this protocol. Moreover, this method has the ability to tolerate a wide variety of substituents in all three components. The results are reproducible and the reactions can be carried out on a gram scale.

Egypt. J. Chem. 56, No.4 (2013) 


\section{Experimental}

Microanalyses were carried out by the Micro Analytical laboratory, National Research Centre, Cairo, Egypt. Infrared spectra ( $\mathrm{KBr}$-disc) were recorded using a Jasco FT/IR-300E spectrometer. ${ }^{1} \mathrm{H}$ NMR and ${ }^{13} \mathrm{C}$ NMR spectra were measured in $\mathrm{CDCl}_{3}$ using Varian Mercury $300 \mathrm{MHz}$ and Varian Gemini $200 \mathrm{MHz}$ with chemical shifts using TMS as standard solvent. Mass spectra were recorded on a GC/MS Finnigan SSQ 7000 spectrometer. All reactions were carried out under atmospheric conditions at room temperature. Tetrachlorosilane (TCS) was obtained from commercial sources. Anhydrous zinc chloride was used as obtained from Aldrich. The solvents were distilled and dried before use. Reactions were monitored by TLC on $0.25 \mathrm{~mm}$ Merck Silica gel sheets (60 GF $354)(4 \times 2 \mathrm{~cm})$ and the spots were detected with UV light.

\section{General procedure}

In a dry two-necked round bottomed flask equipped with a rubber septum, a magnetic stirring bar and a dry condenser, a mixture of diketone $(10 \mathrm{mmol})$, aldehyde (10 mmol), urea (thiourea) $(10 \mathrm{mmol})$, in $\mathrm{CH}_{2} \mathrm{Cl}_{2}(20 \mathrm{ml})$ was allowed to stir with exclusion of moisture at room temperature for $5 \mathrm{~min}$. Tetrachlorosilane $(5 \mathrm{mmol})$ was then added and the mixture was stirred for the specified time as shown in Tables 2 and 3. At the end of the reaction, the mixture was poured onto ice-cold water $(\sim 100 \mathrm{ml})$, neutralized with aq. $\mathrm{Na}_{2} \mathrm{CO}_{3}$, extracted with $\mathrm{CHCl}_{3}(3 \times 30 \mathrm{ml})$ and the extract dried over anhydrous $\mathrm{Na}_{2} \mathrm{SO}_{4}$. The pure solid was filtered, washed with methanol $(3 \mathrm{X} 10 \mathrm{ml})$ and dried under vacuum. This procedure was followed for the preparation of compounds (4) and (5) listed in Tables 2 and 3, respectively. Spectral and analytical data for examples of these products are given below:

5-Ethoxycarbonyl-6- methyl-4- (2-thienyl)-3,4- dihydropyrimidin-2(1H) - thione (4aa)

IR $(\mathrm{KBr}) v\left(\mathrm{~cm}^{-1}\right): 3304,3180,1680,1656,1568 .{ }^{1} \mathrm{H}$ NMR $\delta_{\mathrm{H}}(300 \mathrm{MHz}$, DMSO-d $\left.d_{6}\right): \delta 1.21\left(\mathrm{t}, 3 \mathrm{H}, J=7.0 \mathrm{~Hz}, \mathrm{CH}_{3}\right), 2.32\left(\mathrm{~s}, 3 \mathrm{H}, \mathrm{CH}_{3}\right), 4.12(\mathrm{q}, 2 \mathrm{H}, J=$ $7.0 \mathrm{~Hz}, \mathrm{OCH}_{2} \mathrm{CH}_{3}$ ), 5.49 (d, 1H, $\left.J=10.5 \mathrm{~Hz}, \mathrm{CH}\right), 6.96-7.44$ (m, 3H, ArH) 9.82 (s, 1H, NH), 10.52 (s, 1H, NH). MS (EI $70 \mathrm{ev)} \mathrm{m/z:} 282\left(\mathrm{M}^{+}\right)$. Anal. (\%): Calcd for $\mathrm{C}_{12} \mathrm{H}_{14} \mathrm{~N}_{2} \mathrm{O}_{2} \mathrm{~S}_{2}$ (282.05): C, 51.04; H, 5.00; N, 9.92; S, 22.71 Found: C, 51.00; $\mathrm{H}, 4.94 ; \mathrm{N}, 9.80 ; \mathrm{S}, 22.00$.

5-Acetyl-6-methyl-4-(2-thienyl)-3,4-dihydropyrimidin-2(1H)-thione (4ab)

IR $(\mathrm{KBr}) v\left(\mathrm{~cm}^{-1}\right): 3274,3167,1662,1610,1570 .{ }^{1} \mathrm{H}$ NMR $\delta_{\mathrm{H}}(300 \mathrm{MHz}$, DMSO- $\left.d_{6}\right): \delta 2.21\left(\mathrm{~s}, 3 \mathrm{H}, \mathrm{CH}_{3}\right), 2.31\left(\mathrm{~s}, 3 \mathrm{H}, \mathrm{CH}_{3}\right), 5.30(\mathrm{~d}, 1 \mathrm{H}, J=10.4 \mathrm{~Hz}$, $\mathrm{CH}), 6.94-7.41(\mathrm{~m}, 3 \mathrm{H}, \mathrm{ArH}) 9.85(\mathrm{~s}, 1 \mathrm{H}, \mathrm{NH}), 10.41(\mathrm{~s}, 1 \mathrm{H}, \mathrm{NH}) .{ }^{13} \mathrm{C} \mathrm{NMR}$ (300 MHz, DMSO-d6): $\delta$ 196.30, 184.20, 150.20, 139.3, 126.5, 125, 123.4, 113.52, 53.60, 22.70, 18.22. MS (EI $70 \mathrm{ev}) \mathrm{m} / \mathrm{z}: 252\left(\mathrm{M}^{+}\right)$. Anal. (\%): Calcd for $\mathrm{C}_{11} \mathrm{H}_{12} \mathrm{~N}_{2} \mathrm{OS}_{2}$ (252.04): C, 52.35; H, 4.79; N, 11.10; S, 25.41 Found: C, 52.22; H, $4.74 ; \mathrm{N}, 10.80 ; \mathrm{S}, 25.20$. 
5-Benzoyl-6-methyl-4-(2-thienyl)-3,4-dihydropyrimidin-2(1H)-thione (4ac)

IR $(\mathrm{KBr}) v\left(\mathrm{~cm}^{-1}\right): 3259,3159,1655,1574 .{ }^{1} \mathrm{H}$ NMR $\delta_{\mathrm{H}}(300 \mathrm{MHz}, \mathrm{DMSO}-$ $\left.d_{6}\right): \delta 2.25\left(\mathrm{~s}, 3 \mathrm{H}, \mathrm{CH}_{3}\right), 5.52(\mathrm{~d}, 1 \mathrm{H}, J=10.4 \mathrm{~Hz}, \mathrm{CH}), 6.95-7.97(\mathrm{~m}, 8 \mathrm{H}, \mathrm{ArH})$, 9.81 (s, 1H, NH), 10.50 (s, 1H, NH). MS (EI $70 \mathrm{ev)} \mathrm{m/z:} 314\left(\mathrm{M}^{+}\right)$. Anal. (\%): Calcd for $\mathrm{C}_{16} \mathrm{H}_{14} \mathrm{~N}_{2} \mathrm{OS}_{2}$ (314.05): C, 61.12; H, 4.49; N, 8.91; S, 20.40 Found: C, $61.7 ; \mathrm{H}, 4.41 ; \mathrm{N}, 8.85 ; \mathrm{S}, 20.34$.

5-Ethoxycarbonyl-6-methyl-4-(1-naphthyl)-3,4-dihydropyrimidin-2(1H)-thione (4ba)

IR $(\mathrm{KBr}) v\left(\mathrm{~cm}^{-1}\right): 3300,3178,1685,1648,1580 .{ }^{1} \mathrm{H}$ NMR $\delta_{\mathrm{H}}(300 \mathrm{MHz}$, DMSO-d $\left.d_{6}\right): \delta 0.90\left(\mathrm{t}, 3 \mathrm{H}, J=7.0 \mathrm{~Hz}, \mathrm{CH}_{3}\right), 2.33\left(\mathrm{~s}, 3 \mathrm{H}, \mathrm{CH}_{3}\right), 3.85(\mathrm{q}, 2 \mathrm{H}, J=$ $\left.6.9 \mathrm{~Hz}, \mathrm{OCH}_{2} \mathrm{CH}_{3}\right), 6.04(\mathrm{~s}, 1 \mathrm{H}, \mathrm{CH}), 7.35-8.30(\mathrm{~m}, 7 \mathrm{H}, \mathrm{ArH}) 9.24(\mathrm{~s}, 1 \mathrm{H}, \mathrm{NH})$, $10.21(\mathrm{~s}, 1 \mathrm{H}, \mathrm{NH})$. MS (EI $70 \mathrm{ev}) \mathrm{m} / \mathrm{z}: 326\left(\mathrm{M}^{+}\right)$. Anal. $(\%)$ : Calcd for $\mathrm{C}_{18} \mathrm{H}_{18} \mathrm{~N}_{2} \mathrm{O}_{2} \mathrm{~S}$ (326.11): C, 66.23; H, 5.56; N, 8.58; S, 9.82. Found: C, 66.15; H, $5.50 ; \mathrm{N}, 8.53 ; \mathrm{S}, 9.73$.

5-Benzoyl-6-methyl-4-(1-naphthyl)-3,4-dihydropyrimidin-2(1H)-thione (4bc)

IR $(\mathrm{KBr}) v\left(\mathrm{~cm}^{-1}\right): 3270,3175,1648,1555 .{ }^{1} \mathrm{H}$ NMR $\delta_{\mathrm{H}}(300 \mathrm{MHz}, \mathrm{DMSO}-$ $\left.d_{6}\right): \delta 2.55\left(\mathrm{~s}, 3 \mathrm{H}, \mathrm{CH}_{3}\right), 5.50(\mathrm{~s}, 1 \mathrm{H}, \mathrm{CH}), 7.08-8.66(\mathrm{~m}, 12 \mathrm{H}, \mathrm{ArH}), 9.34(\mathrm{~s}, 1 \mathrm{H}$, $\mathrm{NH}), 10.05(\mathrm{~s}, 1 \mathrm{H}, \mathrm{NH})$. MS (EI $70 \mathrm{ev}) \mathrm{m} / \mathrm{z}: 357\left(\mathrm{M}^{+}\right)$. Anal. (\%): Calcd for $\mathrm{C}_{22} \mathrm{H}_{18} \mathrm{~N}_{2} \mathrm{O}_{2}$ (358.11): C, 73.71; H, 5.06; N, 7.82; S, 8.95. Found: C, 73.66; H, $5.01 ; \mathrm{N}, 7.75 ; \mathrm{S}, 8.87$.

5-Acetyl-6-methyl-4-(2-thienyl)-3,4-dihydro-1H-pyrimidin-2-one (5ab)

IR $(\mathrm{KBr}) v\left(\mathrm{~cm}^{-1}\right): 3278,3169,1666,1610,1575 .{ }^{1} \mathrm{H}$ NMR $\delta_{\mathrm{H}}(300 \mathrm{MHz}$, DMSO- $\left.d_{6}\right): \delta 2.22\left(\mathrm{~s}, 3 \mathrm{H}, \mathrm{CH}_{3}\right), 2.33\left(\mathrm{~s}, 3 \mathrm{H}, \mathrm{CH}_{3}\right), 5.30(\mathrm{~d}, 1 \mathrm{H}, J=10.2 \mathrm{~Hz}$, $\mathrm{CH}), 6.92-7.50(\mathrm{~m}, 3 \mathrm{H}, \mathrm{ArH}) 9.88(\mathrm{~s}, 1 \mathrm{H}, \mathrm{NH}), 10.56$ (s, 1H, NH). MS (EI $70 \mathrm{ev})$ $\mathrm{m} / \mathrm{z}$ : $236\left(\mathrm{M}^{+}\right)$. Anal. (\%): Calcd for $\mathrm{C}_{11} \mathrm{H}_{12} \mathrm{~N}_{2} \mathrm{OS}_{2}$ (236.29): C, 55.91; H, 5.12; N, 11.86; S, 13.57 Found: C, 55.86; H, 5.08; N, 11.82; S, 13.52.

4(1-Naphthyl)- 3,4-hydro-1H-indeno[1,2-d]pyrimidin-2,5-dione (5bd)

IR $(\mathrm{KBr}) v\left(\mathrm{~cm}^{-1}\right): 3392,3207,1671,1613 .{ }^{1} \mathrm{H}$ NMR $\delta_{\mathrm{H}}(300 \mathrm{MHz}, \mathrm{DMSO}-$ $\left.d_{6}\right): \delta 5.79(\mathrm{~s}, 1 \mathrm{H}, \mathrm{CH}), 7.14-8.32(\mathrm{~m}, 11 \mathrm{H}, \mathrm{ArH}), 9.57(\mathrm{~s}, 1 \mathrm{H}, \mathrm{NH}), 10.07(\mathrm{~s}, 1 \mathrm{H}$, NH). MS (EI $70 \mathrm{ev)} \mathrm{m/z:} 326\left(\mathrm{M}^{+}\right)$. Anal. (\%): Calcd for $\mathrm{C}_{21} \mathrm{H}_{16} \mathrm{~N}_{2} \mathrm{O}$ (326.11): C, 77.29; H, 4.32; N, 8.58. Found: C, 77.25; H, 4.26; N, 8.49.

\section{References}

1. Kappe, C.O., 100 years of the Biginelli dihydropyridine synthesis.Tetrahedron, 49, 6937 (1993); (b) Kappe, C.O., Recent advances in the Biginelli dihydropyrimidine synthesis, new tricks from an old dog. Chem. Res. 33, 879 (2000).

2. Hu, E.H., Sidler, D. R. and Dolling, U.H., Unprecedented catalytic three component one-pot condensation reaction: An efficient synthesis of 5-Alkoxycarbonyl- 4-aryl3,4-dihydropyrimidin-2(1H)-ones. J. Org. Chem. 63, 3454 (1998).

3. Kappe, C.O. and Falsone, S.F., Synthesis and reactions of biginelli compounds, 12 . polyphosphate ester-mediated synthesis of dihydropyrimidines, improved conditions for the Biginelli reaction. Synlett. 718 (1998).

Egypt. J. Chem. 56, No.4 (2013) 
4. Bigi, F., Carloni, S., Frullanti, B., Maggi, R. and Sartori, G., A revision of the Biginelli reaction under solid acid catalysis. Solvent-free synthesis of dihydropyrimidines over montmorillonite KSF. Tetrahedron Lett. 40, 3465 (1999).

5. Singh, K., Singh, J., Deb, P.K. and Singh, H., An expedient protocol of the Biginelli dihydropyrimidine synthesis using carbonyl equivalents. Tetrahedron, 55, 12873 (1999).

6. Lu, J. and Ma, H., Iron(III)-catalyzed synthesis of dihydropyrimidinones. Improved conditions for the Biginelli reaction. Synlett, 63 (2000).

7. (a) O'Reilly, B.C. and Atwal, K.S., Synthesis of substituted 1,2,3,4-tetrahydro-6methyl-2-oxo-5-pyrimidinecarboxylic acid esters: The Biginelli condensation. Heterocycles, 26, 1185 (1987); (b) Atwal, K.S., O'Reilly, B.C., Gougoutas, J.Z. and Malley, M.F., Synthesis of substituted 1,2,3,4-tetrahydro-6methyl-2-thioxo-5-pyrimidinecarboxylic acid esters. Heterocycles, 26, 1189 (1987); (c) Atwal, K.S., Rovnyak, G. C., O'Reilly, B. C. and Schwartz, J., Substituted 1,4dihydropyrimidines. 3. Synthesis of selectively functionalized 2-hetero-1,4dihydropyrimidines. J. Org. Chem. 54, 5898 (1989); (d) Kappe, C.O., Kumar, D. and Varma, R.S., Microwave-assisted high-speed parallel synthesis of 4-aryl-3,4dihydropyrimidin-2 $(1 H)$-ones using a solventless Biginelli condensation protocol. Synthesis, 1799 (1999).

8. Yadav, J.S., Subba, B.V., Reddy, K.B., Raj, K.S. and Prasad, A.R., Ultrasoundaccelerated synthesis of 3,4-dihydropyrimidin-2(1H)-ones with ceric ammonium nitrate. J. Chem. Soc., Perkin Trans, 1, 1939 (2001).

9. Ma, Y., Qian, C., Wang, L. and Yang, M., Lanthanide triflate catalyzed Biginelli reaction. one-pot synthesis of dihydropyrimidinones under solvent-free conditions. $J$. Org. Chem. 65, 3864 (2000).

10. Ranu, B.C., Hajra, A. and Jana, U., Indium(III) chloride catalyzed one-pot synthesis of dihydropyrimidinones by a three-component coupling of 1,3-dicarbonyl compounds, aldehydes, and urea: An improved procedure for the Biginelli reaction. $J$. Org. Chem. 65, 6270 (2000).

11. Lu, J., Bai, Y., Wang, Z., Yang, B. and Ma, H., One-pot synthesis of 3,4dihydropyrimidin-2 $(1 H)$-ones using lanthanum chloride as a catalyst. Tetrahedron Lett. 41, 9075 (2000).

12. Fu, N.Y., Yuan, Y.F., Cao, Z., Wang, S.W., Wang, J.T. and Peppe, C., Indium (III) bromide-catalyzed preparation of dihydropyrimidinones: improved protocol conditions for the Biginelli reaction. Tetrahedron, 58, 4801(2002).

13. Kumar, K.A., Kasthuraiah, M., Reddy, C.S. and Reddy, C.D., $\mathrm{Mn}(\mathrm{OAc})_{3} \cdot 2 \mathrm{H}_{2} \mathrm{O}-$ mediated three-component, one-pot,condensation reaction: an efficient synthesis of 4 aryl-substituted3,4-dihydropyrimidin-2-ones. Tetrahedron Lett. 42, 7873 (2001). 
14. Kappe, C.O., Kumar, D. and Varma, R.S., Microwave-assisted high-speed parallel synthesis of 4-Aryl-3,4-dihydropyrimidin-2(1H)-ones using a solventless Biginelli condensation protocol. Synthesis, 1799 (1999).

15. Maradur, S.P. and Gokavi, G.S., Heteropoly acid catalyzed synthesis of 3,4dihydropyrimidin-2(1H)-ones. Catalysis Commun. 8(3), 279 (2007).

16. Singh, K., Arora, D. and Singh, S., Dowex-promoted general synthesis of N,N $\square$ disubstituted-4-aryl-3,4-dihydropyrimidinones using a solvent-free Biginelli condensation protocol. Tetrahedron. Lett. 47(25), 4205 (2006).

17. Afiee, E. and Shahbazi, F., One-pot synthesis of dihydropyrimidones using silicasupported heteropoly acid as an efficient and reusable catalyst: improved protocol conditions for the Biginelli reaction. Journal of Molecular Catalysis A, 250 (1-2), 57 (2006).

18. Salehi, H. and Guo, Q.X., A facile and efficient one-pot synthesis of dihydropyrimidinones catalyzed by magnesium bromide under solvent-free conditions. Synthetic Commun. 34(1), 171 (2004).

19. Lei, M., Wu, D.D., Wei, H.G. and Wang, Y., Polymer-supported 4aminoformoyldiphenylammonium triflate (PS-AFDPAT): an effective and recyclable catalyst for the Biginelli reaction. Synthetic Commun. 39(3), 475 (2009).

20. Chari, M.A. and Syamasundar, K., Silica gel supported sodium hydrogensulfate as a heterogeneous catalyst for high yield synthesis of 3,4-dihydropyrimidin-2 $(1 \mathrm{H})$-ones. Journal of Molecular Catalysis A, 221(1-2), 137 (2004).

21. Lu, J. and Bai, Y., Catalysis of the Biginelli reaction by ferric and nickel chloride hexahydrates. One-pot synthesis of 3,4-dihydropyrimidin-2(1H)-ones. Synthesis, 4, 466 (2002).

22. Reddy, C.V., Mahesh, M., Raju, P.V.K., Babu, T.R. and Reddy, V.V.N., Zirconium(IV) chloride catalyzed one-pot synthesis of 3,4-dihydropyrimidin-2(1H)ones. Tetrahedron Lett. 43(14), 2657 (2002).

23. Paraskar, A.S., Dewkar, G.K. and Sudalai, A., $\mathrm{Cu}(\mathrm{OTf}) 2$ : a reusable catalyst for high-yield synthesis of 3,4-dihydropyrimidin-2(1H)-ones. Tetrahedron Lett. 44(16), 3305 (2003).

24. Varala, R., Alam, M.M. and Adapa, S.R., Bismuth triflate catalyzed one-pot synthesis of 3,4-dihydropyrimidin-2 (1H)-ones: an improved protocol for the Biginelli reaction. Synlett. 1, 67 (2003).

25. Ma, Y., Qian, C. and Yang, M., Lanthanide triflate catalyzed Biginelli reaction. Onepot synthesis of dihydropyrimidinones under solvent-free conditions. The Journal of Organic Chemistry, 65(12), 3864 (2000).

26. Kotharkar, S.A., Jadhav, M.R., Nagawade, R.R., Bahekar, S.S. and Shinde, D.B., Sulphamic acid $\left(\mathrm{H}_{2} \mathrm{NSO}_{3} \mathrm{H}\right)$ catalysed one potsynthesis of 3,4-dihydropyrimidin-2(1H) ones and their thione analogues. Letters in Organic Chemistry, 2, 662 (2005).

Egypt. J. Chem. 56, No.4 (2013) 
27. Heravi, M.M., Bakhtiari, K. and Bamoharram, F.F., 12-Molybdophosphoric acid: a recyclable catalyst for the synthesis of Biginelli-type 3,4-dihydropyrimidine-2(1H)ones. Catalysis Commun. 7(6), 373 (2006).

28. Tajbakhsh, M., Mohajerani, B., Heravi, M.M. and Ahmadi, A. N., Natural HEU type zeolite catalyzed Biginelli reaction for the synthesis of 3,4-dihydropyrimidin2(1H) one derivatives. Journal of Molecular Catalysis A, 236(1-2), 216 (2005).

29. Su, W., Li, J., Zheng, Z. and Shen, Y., One-pot synthesis of dihydropyrimidiones catalyzed by strontium(II)triflate under solvent-free conditions. Tetrahedron Lett. 46(36), 6037 (2005).

30. Gupta, R. and Paul, S., Covalently anchored sulfonic acid onto silica as an efficient and recoverable interphase catalyst for the synthesis of 3,4-dihydropyrimidinones/ thiones. Journal of Molecular Catalysis A, 266(1-2), 50 (2006).

31. Shirini, F., Zolfigol, M. A. and Mollarazi, E., $\mathrm{ZrOCl}_{2} .8 \mathrm{H}_{2} \mathrm{O}$ as an efficient reagent for the solvent-free synthesis of 3,4-dihydropyrimidin-2-(1H)-ones. Synthetic Commun. 36(16), 2307 (2006).

32. Shirini, F., Marjani, K. and Nahzomi, H. T., Silica triflate as an efficient catalyst for the solvent-free synthesis of 3,4-dihydropyrimidin-2(1H)-ones. Arkivoc, 1, 51 (2007).

33. Shirini, F., Zolfigol M. A. and Abri, A. R., $\mathrm{Fe}\left(\mathrm{HSO}_{4}\right)_{3}$ as an efficient catalyst for the preparation of 3,4-dihydropyrimidin-2(1H)-ones in solution and under solvent-free conditions. Journal of the Iranian Chemical Society, 5(1), 96 (2008).

34. Shirini, F., Zolfigol M. A. and Mollarazi, E., Solvent-free synthesis of 3,4dihydropyrimidin-2(1H)-ones using trichloroisocyanuric acid. Letters in Organic Chemistry, 2(8), 718 (2005).

35. Debache, A., Amimour, M., Belfaitah, A., Rhouati, S. and Carboni, B., A one-pot Biginelli synthesis of 3,4-dihydropyrimidin-2-(1H)-ones/thiones catalyzed by triphenylphosphine as Lewis base. Tetrahedron Lett. 49(42), 6119 (2008).

36. Chitra, S. and Pandiarajan, K., Calcium fluoride: an efficient and reusable catalyst for the synthesis of 3,4-dihydropyrimidin-2(1H)-ones and their corresponding 2(1H)thione: an improved high yielding protocol for the Biginelli reaction.Tetrahedron Lett. 50(19), 2222 (2009).

37. Jain, S.L., Joseph, J.K. and Sain, B., Ionic liquid promoted an improved synthesis of 3,4-dihydropyrimidinones using[bmim] BF4 immobilized $\mathrm{Cu}$ (II) acetylacetonate as recyclable catalytic system. Catalysis Lett. 115(1-2), 52 (2007).

38. Chen, X. and Peng, Y., Chloroferrate(III) ionic liquid: efficient and recyclable catalyst for solvent-free synthesis of 3,4-dihydropyrimidin-2(1H)-ones. Catalysis Lett. 122(3-4), 310 (2008).

39. Dadhania, A.N., Patel, V.K. and Raval, D.K., A convenient and efficient protocol for the one pot synthesis of 3,4-dihydropyrimidin-2-(1H)-ones catalyzed by ionic liquids under ultrasound irradiation. The Journal of the Brazilian Chemical Society, 22(3), 511 (2011). 
40. Shirini, F., Zolfigol, M.A. and Albadi, J., Melamine trisulfonic acid: a new, efficient and recyclable catalyst for the synthesis of 3,4-dihydropyrimidin-2(1H)-ones/thiones in the absence of solvent. Chinese Chemical Letters, 22(3), 318 (2011).

41. (a) Zolfigol, M.A., Silica sulfuric acid/ $/ \mathrm{NaNO}_{2}$ as a novel heterogeneous system for production of thionitrites and disulfides under mild conditions. Tetrahedron, 57, 9509 (2001); (b) Mirjalili, B.F., Zolfigol, M. A. and Bamoniri, A., Deprotection of acetals and ketals by silica sulfuric acid and wet $\mathrm{SiO}_{2}$. Molecules, 7, 751 (2002).

42. Kappe, C.O., Kumar, D. and Varma, R.S., Microwave assisted high speed parallel synthesis of 4-aryl-3,4-dihydropyrimidin-2(1H)-ones using a solventless Biginelli condensation protocol. Synthesis, 1799 (1999).

43. (a) Atwal, K.S., Rovnyak, G.C., O'Reilly, B.C. and Schwartz, J., Substituted synthesis of selectively functionalized 2-hetero-1,4-dihydropyrimidines. J. Org. Chem. 54, 5898 (1989); (b) Atwal, K.S., Swanson, B.N., Unger, S.E., Floyd, D.M., Moreland, S., Hedberg, A. and O'Reilly, B.C., Dihydropyrimidine calcium channel blockers.3.3. carbamoyl-4-aryl-1,2,3,4-tetrahydro-6-methyl-5pyrimidinecarboxylic acid esters as orally effective antihypertensive. agents. J. Med. Chem. 34, 806 (1991); (c) Rovnyak, G.C., Atwal, K. S., Hedberg, A., Kimball, S.D., Moreland, S., Gougoutas, J.Z., O'Reilly, B.C., Schwartz, J. and Malley, M.F., Dihydropyrimidine calcium channel blockers. 4. Basic 3-substituted-4-aryl-1,4dihydropyrimidine-5-carboxylic acid esters. Potent antihypertensive agents, Conformational analysis of 4-aryl-dihydropyrimidine calcium channel modulators. A comparison of ab initio, semiempirical and X-ray crystallographic studies. J. Med. Chem. 35, 3254 (1992).

44. (a) Patil, A.D., Kumar, N.V., Kokke, W.C., Bean, M.F., Freyer, A.J., DeBrosse, C., Mai, S., Truneh, A. and Faulkner, D.J., Novel alkaloids from the sponge batzella sp.: Inhibitors of HIV gp120-Human CD4 binding. J. Org. Chem. 60, 1182 (1995); (b) Snider, B.B., Chen, J., Patil, A.D. and Freyer, A., Synthesis of the tricyclic portions of batzelladines A, B and D. Revision of the stereochemistry of batzelladines A and D. Tetrahedron Lett., 37, 6977 (1996); (c) Rama Rao, R.V., Gurjar, M.K. and Vasudevan, J., An enantiospecific synthesis of the tricyclic guanidine segment of the anti-HIV marine alkaloid batzelladine A. J. Chem. Soc. Chem. Commun. 1369 (1995).

45. Sergey, V.R., Andrey, S.P., Eugeniy, N.O., Dmitriy, M.V., Oleg, V.S. and Andrey, A.T., $\mathrm{CF}_{3}$-substituted 1,3-dicarbonyl compounds in the Biginelli reaction promoted by chlorotrimethylsilane. Journal of Fluorine Chemistry, 129(7), 625 (2008).

46. Hong, W.Z., Jin, X.W. and Xi, T.W., Solvent- and catalyst-free synthesis of dihydropyrimidinthiones in one-pot under focused microwave irradiation conditions. Chinese Chemical Letters, 19(10),1183 (2008).

47. Zitao, W., Yang, W., Wen-Xiong, Z., Zhaomin, H., and Zhenfeng, X., Efficient one-pot synthesis of 2,3-dihydropyrimidinthiones via multicomponent coupling of terminal alkynes. Elemental sulfur and carbodiimides. Am. Chem. Soc. 131 (42), 15108 (2009).

48. Jean, C. L., Jean, J.V.E., Loic, T. and Jean, P.B., A three-component condensation protocol based on ionic liquid phase bound acetoacetate for the synthesis of Biginelli 3,4-dihydropyrimidine-2(1H)-ones. Arkivoc, (iii) 13 (2007).

Egypt. J. Chem. 56, No.4 (2013) 
49. Chitra, S. and Devanathan, D., Synthesis and in vitro microbiological evaluation of 5-acetyl-4-aryl-6-methyl-3,4-dihydropyrimidin-2 $(1 H)$-thiones using calcium fluoride as catalyst. International Journal of Pharmaceutical and Biological Research (IJPBR), 3(4), 149 (2012).

50. Hattori, K., Kohchi, Y., Oikawa, N., Suda, H., Ura, M., Ishikawa, T., Miwa, M., Endoh, M., Eda, H., Tanimura, H., Kawashima, A., Horii, I., Ishitsuka, H. and Shimma, N., Design and synthesis of the tumor-activated prodrug of dihydropyrimidine dehydrogenase (DPD) inhibitor, RO0094889 for combination therapy with capecitabine. Bioorg. Med. Chem. Lett. 13, 867 (2003).

51. Phucho, I.T., Nongpiur, A., Tumtin, S., Nongrum, R. and Nongkhlaw, R.L., Recent progress in the chemistry of dihydropyrimidinones. Rasayan J. Chem. 2(3), 662 (2009).

52. Salehi, P., Dabiri, M., Zolfigol, M.A. and Bodaghi Fard, M.A., Efficient synthesis of 3,4-dihydropyrimidin-2(1H)-ones over silica sulfuric acid as areusable catalyst under solvent-free conditions. Heterocycles, 60, 2435 (2003).

53. Nasr-Esfahani, M., Montazerozohori, M. and Abdi, K., Photocatalytic oxidation of dihydropyrimidinones using titanium dioxide suspension. Arkivoc, (x), 255 (2009).

54. (a) Elmorsy, S.S., Badawy, D.S. and Khatab, T.K., Evaluation of TCS/ZnCl $\mathrm{Zn}_{2}$ with acetic anhydride as an acetylating reagent for methylene ketones. Phosphorus, Sulfur. Silicon \& Relat. Elem., 180, 109 (2005); (b) Elmorsy, S.S., Badawy, D.S. and Khatab, T.K., Phosphorus, Sulfur, Silicon \& Relat. Elem. 181, 2005 (2006); (c) Salama, T.A., Elmorsy, S.S., Khalil, A.M. and Ismail, M.A., A $\mathrm{SiCl}_{4}$ $\mathrm{ZnCl}_{2}$ induced general, mild and efficient one-pot, three-component synthesis of $\beta$ amido ketone libraries. Tetrahedron Lett. 48, 6199 (2007); (d) Badawy, D.S., AbdelGalil, E., Kandeel, E.M., Basyouni, W.M., El-Bayouki K.A.M. and Khatab, T.K., Synthesis and antioxidant and antitumor activity of novel pyridine, chromene, thiophene and thiazole derivatives. Phosphorus Sulfur, Silicon Relat. Elem. 184, 220 (2009); (e) Badawy, D.S., Abdel-Galil, E., Kandeel, E.M., Basyouni, W.M. and Khatab, T.K., An efficient synthesis of $\beta$-acylureas via a three-component, onepot synthesis. Phosphorus, Sulfur, Silicon Relat. Elem. 184, 2799 (2009); (f) Salama, T.A., El-Ahl, A.-A.S., Elmorsy, S.S., Khalil, A.M. and Ismail, M.A., Tetrahedron Lett. 50, 5933 (2009); (g) Khatab, T.K., El-Bayouki, K.A.M. and Basyouni, W.M., An efficient synthesis of $\beta$-acylureas via a three-component, one-pot synthesis using $\mathrm{TCS} / \mathrm{ZnCl}_{2}$. Tetrahedron Lett. 52, 1448 (2011).

55. (a) Shah, V.R., Godhasra. J.N., Patel, M.C. and Kansagara, N.N., Microwave assisted direct rabid and efficient synthesis of some novel dihydropyrimidines and evaluation of their antimicrobial activities. Int. J. Chem. Sci. 7(3), 1575 (2009); (b) Heys, L., Moore, C.G. and Murphy, P.J., The guanidine metabolites of Ptilocaulisspiculifer and related compounds; isolation and synthesis. Chem. Soc. Rev. 29, 57 (2000).

56. Hitendra, N.K., Manisha, S. and Kaushik, M.P., Synthesis of 4-aryl substituted 3,4dihydropyrimidinones using silica-chloride under solvent free conditions. Molecules, 12, 1341 (2007).

57. Chennan, R. and Young-Woo, K., Tetrahedron, 64, 5023 (2008). 
58. Nan-Yan, F., Yao-Feng, Y., Zhong, C., Shan-Wei, W., Ji-Tao, W. and Clovis, P., Indium (III) bromide-catalyzed preparation of dihydropyrimidinones: improved protocol conditions for the Biginelli reaction. Tetrahedron, 58, 4801 (2002).

59. Juan, C.R.D., Dan, B. and Gilbert, K., $\mathrm{ZrCl}_{4}$ or $\mathrm{ZrOCl}_{2}$ under neat conditions: optimized green alternatives for the Biginelli reaction. Tetrahedron Lett. 48, 5777 (2007).

60. Jhillu, S.Y., Basi, V.S.R., Srinivas, R., Venugopal, C. and Ramalingam, T., $\mathrm{LiClO}_{4}$-catalyzed one-pot synthesis of dihydropyrimidinones: an improved protocol for Biginelli reaction. Synthesis, 9, 1341 (2001).

61. Uttara, S., Varadaraj, G.B., Sidhartha S.K. and Jesil, A.M.M., Mimic Biginelli dihydropyrimidinone derivatives: Synthesis, cytotoxicity screening against HepG2 and HeLa cell lines and molecular modeling study. Organic and Medicinal Chemistry Letters, 2-11 (2012).

62. Sami, S., Eshagh, R.N. and Ghasem D., 1-Methyl-3-(2-(sulfooxy)ethyl)-1Himidazol-3-ium chloride as a new and green ionic liquid catalyst for one-pot synthesis of dihydropyrimidinones under solvent-free condition. Journal of Chemistry, 1-6, http://dx.doi.org/10.1155/2013/834656 (2013).

(Received 6/11/2013;

accepted 2/12/2013) 
طريقة فعالة لتثييد مشتقات البيرميدينون والبيرميدينتيون الواعدة

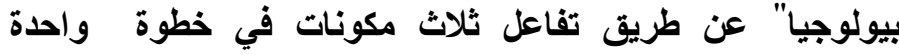
باستخدام حفاز رابع كلوريد السيليكون تلاعن

تامر كمال خطاب، خيري عبد الحميد البيوقي ، وحيد محمد بسيوني و فريد محمد

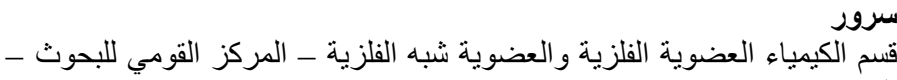

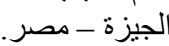

تم تثبيد سلسلة من مشتقات البير ميدينون و البير ميدينثيون بناتج نهائي عالي النسبة

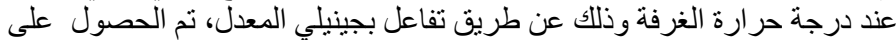

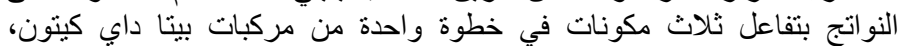

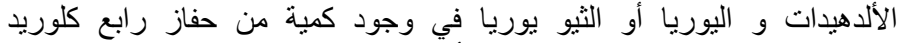

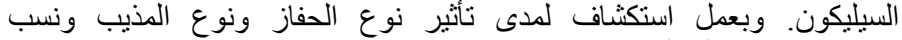

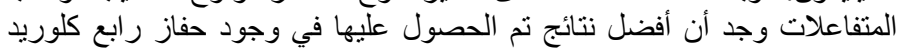

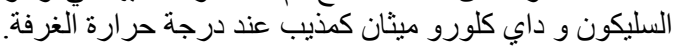

